\title{
Learning from Label Preferences ${ }^{\star}$
}

\author{
Eyke Hüllermeier ${ }^{1}$ and Johannes Fürnkranz ${ }^{2}$ \\ 1 Philipps-Universität Marburg, Germany \\ 2 Technische Universität Darmstadt, Germany
}

\begin{abstract}
In this paper, we review the framework of learning (from) label preferences, a particular instance of preference learning. Following an introduction to the learning setting, we particularly focus on our own work, which addresses this problem via the learning by pairwise comparison paradigm. From a machine learning point of view, learning by pairwise comparison is especially appealing as it decomposes a possibly complex prediction problem into a certain number of learning problems of the simplest type, namely binary classification. We also discuss how a number of common machine learning tasks, such as multi-label classification, hierarchical classification or ordinal classification, may be addressed within the framework of learning from label preferences. We also briefly address theoretical questions as well as algorithmic and complexity issues.
\end{abstract}

Preference learning is a recent addition to the suite of learning tasks in machine learning [1, where the training information is typically not given in the form of scalar outputs, but instead in the form of pairwise comparisons expressing preferences between different objects. One can distinguish learning from object preferences, where the training data is given in the form of pairwise comparisons between objects, and learning from label preferences, where the training data is given in the form of pairwise comparisons between labels that are attached to the objects. In the former case, a common performance task is to rank a new set of objects (object ranking), whereas in the latter case, the performance task is to rank the set of labels for a new object (label ranking). Besides, the training information may also be given in the form of (ordinal) preference degrees attached to the objects, indicating an absolute (as opposed to a relative/comparative) assessment. If the task is to rank a new set of objects according to their preference degrees, we also speak of instance ranking. In this talk, we focus on the task of label ranking, and, in particular, on our own work in this area, which concentrates on learning a set of pairwise comparators (LPC) [2].

\section{References}

1. Fürnkranz, J., Hüllermeier, E. (eds.): Preference Learning. Springer, Heidelberg (2010)

2. Fürnkranz, J., Hüllermeier, E.: Preference learning and ranking by pairwise comparison. In: [1], pp. 65-82

* The full version of this paper is published in the Proceedings of the 14th International
Conference on Discovery Science, Lecture Notes in Artificial Intelligence Vol. 6926 .

J. Kivinen et al. (Eds.): ALT 2011, LNAI 6925, p. 38, 2011.

(C) Springer-Verlag Berlin Heidelberg 2011 